\title{
Eosinopenia Predicting Long-Term Mortality In Hospitalized Acute Exacerbation of COPD Patients With Community-Acquired Pneumonia - A Retrospective Analysis
}

\section{Yanxiong Mao}

Key Laboratory of Respiratory Disease of Zhejiang Province, Department of Respiratory and Critical Care Medicine, Second Affiliated Hospital of Zhejiang University School of Medicine, Hangzhou

\section{Yuanyuan Qian}

Department of Respiratory and Critical Care Medicine, Haining People's Hospital, Haining Branch, The First Affiliated Hospital, Zhejiang University, Haining

\section{Xiaoyan Sun}

Women's Hospital, School of Medicine, Zhejiang University

$\mathrm{Na} \mathrm{Li}$

Key Laboratory of Respiratory Disease of Zhejiang Province, Department of Respiratory and Critical Care Medicine, Second Affiliated Hospital of Zhejiang University School of Medicine, Hangzhou Huaqiong Huang ( $\nabla$ zr_hhq@zju.edu.cn )

Key Laboratory of Respiratory Disease of Zhejiang Province, Department of Respiratory and Critical Care Medicine, Second Affiliated Hospital of Zhejiang University School of Medicine, Hangzhou

\section{Research Article}

Keywords: eosinopenia, chronic obstructive pulmonary disease, community-acquired pneumonia, mortality

Posted Date: July 8th, 2021

DOl: https://doi.org/10.21203/rs.3.rs-650145/v1

License: (1) This work is licensed under a Creative Commons Attribution 4.0 International License. Read Full License

Version of Record: A version of this preprint was published at International Journal of Chronic Obstructive Pulmonary Disease on December 1st, 2021. See the published version at https://doi.org/10.2147/COPD.S347948. 


\section{Abstract}

Background: Chronic obstructive pulmonary disease (COPD) and community-acquired pneumonia (CAP) are both common respiratory diseases. Acute exacerbation of COPD (AECOPD) could be triggered by CAP. Peripheral blood eosinopenia are strongly associated with increased mortality. In hospitalized AECOPD patients with CAP, eosinopenia may be used to identified patients with high risk of death on admission.

Methods: We conducted a retrospective cohort study of hospitalized AECOPD patients with CAP. Patients who had received systemic corticosteroids preadmission were excluded. The patients were identified by individual case file review. According to blood eosinophil count, patients were grouped as eosinopenia $(<50 / \mu \mathrm{L})$ and non-eosinopenia $(\geq 50 / \mu \mathrm{L})$. Associations of eosinopenia with infection and 18month survival were analyzed using appropriate statistical methods.

Results: Baseline demographic, comorbidity and CURB65 score were similar between two groups. The eosinopenia group had significantly higher pro-BNP, D-dimer, neutrophil percentage, and lower lymphocyte count and lymphocyte percentage. The eosinopenia group had significantly higher CRP (median 77.30 vs $16.55, p=0.008$ ) and procalcitonin (median 0.32 vs $0.12, p=0.001$ ). Survival at 18 months after hospital discharge was significantly lower in the eosinopenia group vs non-eosinopenia group (log rank, $p=0.002)$

Conclusions: Eosinopenia $(<50 / \mu \mathrm{L})$ was a strong predictor of 18 -month mortality, and associated with more severe infection in hospitalized AECOPD patients with CAP. Eosinophil count at admission can be used as a prognosis marker of mortality in those population.

\section{Background}

Chronic obstructive pulmonary disease (COPD) is a common respiratory disease worldwide. (1) Acute exacerbations of chronic obstructive pulmonary disease (AECOPD) are important causes of hospital admission and mortality.(2) Community-acquired pneumonia (CAP) is another common respiratory disease, and occurs commonly in patients with COPD. It is estimated that there is an approximately 18fold greater incidence of CAP in COPD patients than in those without COPD. (3) COPD is also frequent among CAP patients. COPD as a comorbidity is reported in $35-50 \%$ of hospitalized patients with CAP. (4) The CAP and AECOPD come together when COPD patients acquire AECOPD triggered by CAP. The clinical presentations of these episodes meet the accepted criteria for the diagnosis of AECOPD and CAP in which a pulmonary infiltrate is found by chest radiograph.

Eosinopenia, defined as a reduced eosinophil count in peripheral blood, could be caused by either acute infection or acute stress. (5) Eosinopenia is reported to be a useful predictor of bloodstream infection or sepsis. $(6,7)$ Eosinopenia is an early marker of increased mortality in Intensive Care Unit (ICU)patients.(5, 8) Eosinopenia in AECOPD was also concluded to have some clinical implications in published studies. In AECOPD patients, an inverse relationship between bacterial infection and eosinophil count has been reported.(9) The AECOPD patients with bacterial infection had a significant decrease in the blood 
eosinophil count compared to the stable state. Eosinopenia is also an independent predictor of mortality and length of stay in AECOPD patients.(10-12)

The number of published articles on AECOPD with CAP was relatively small, compared to articles on AECOPD or CAP. The potential utility of using eosinopenia to guide optimal management of AECOPD with CAP has not been explored. The aim of the present study was to evaluate association between eosinopenia and clinical outcomes in hospitalized AECOPD patients with CAP.

\section{Methods}

The present study was a retrospective cohort study of hospitalized AECOPD patients with CAP at an academic teaching tertiary care hospital (Second Affiliated Hospital of Zhejiang University School of Medicine, China). Ethical approval was sought and granted by Hospital Ethics Committee.

\section{Patient selection}

All patients admitted to the study hospital between Jan 2019 and June 2019 with discharge diagnosis of both AECOPD and CAP were retrieved from Electric Medical Record System(EMRS). Patients who had received systemic corticosteroids preadmission were identified from medical record and excluded from study.

\section{Confirmation of diagnosis}

The definition of AECOPD was based on baseline clinical data obtained by chart review as described before. (13) This strategy is widely used in the application of prognostic prediction rules and reflects the methods used in the original pneumonia severity index (PSI) score studies.(14)

Records were inspected by a pulmonologist (Y.Y.Q), who was kept blinded to eosinophil designation, to confirm CAP. In Chinese tertiary care hospitals, it is a common practice to order a chest computed tomography (CT) for patients suspected of CAP. So the diagnosis of CAP in this study were all based on chest CT. Patients were defined as having CAP as described by the Chinese CAP guideline in adults, (15) when the following four criteria were met: (1) onset in community.; (2) at least one of the followings (a) new onset of cough or expectoration, or aggravation of existing symptoms of respiratory tract diseases, with or without purulent sputum, chest pain, dyspnea, or hemoptysis (b) fever $>37.5^{\circ} \mathrm{C}$, (c) signs of pulmonary consolidation and/or moist rales (d) peripheral white blood cell (WBC) count $>10 \times 10^{9} / \mathrm{L}$ or $<4 \times 10^{9} / \mathrm{L}$, with or without a left shift; (3) chest CT scan at the time of hospitalization showing new patchy infiltrates, lobar or segmental consolidation, ground-glass opacities, or interstitial changes, with or without pleural effusion, defined by a radiologist's reading; and (4) no alternative diagnosis at the time of hospital discharge that justified the presence of criteria 2 and 3.

\section{Data collection}


Demographic data, lab test results on admission, disease comorbidities, and pharmacotherapy were collected from EMRS. The eosinophil count from the first whole blood cell count obtained in the hospital was used to designate eosinophil group. In accordance with previous studies, we defined eosinopenia as $<50 / \mu \mathrm{L}$ and non-eosinopenia as $\geq 50 / \mu \mathrm{L}$. (12) Mortality status was assessed by phone call during early June 2021.

\section{Data Analysis}

The results were analyzed using IBM SPSS Statistics 20. Continuous data was presented as the mean with stand deviation (SD)or median with interquartile range (IQR), depending on the distribution of data. Variables were compared using the unpaired Student's t-test, Welch t-test or the Wilcoxon rank sum test with continuity correction, depending on data normality and homogeneity of variance. Categorical data were presented as absolute value and percentage, and analyzed using Chi-square test or Fisher's exact test according to test assumptions. Statistical significance was set at $p<0.05$.

\section{Results}

A total number of 82 admissions with discharge diagnosis of both AECOPD and CAP were identified from EMRS. After review case by case, 59 admissions of AECOPD with CAP were confirmed and eligible for further analysis (Figure 1). The eosinopenia group had 41 admissions, and the non-eosinopenia group had 18 admissions.

\section{Demographic, comorbidity and clinical expression}

Baseline demographic and comorbidity data were similar between two groups (Table 1). The proportion of patients with atrial fibrillation was higher in the eosinopenia group, but without statistical significance. The CURB65 score was not different between two groups, which indicated that the severity of CAP between two groups was similar (Table 2). Compared to the non-eosinopenia group, the eosinopenia group had a higher proportion of patients admitted via Emergency Department (ED) (97.6\% vs $77.8 \%, p=0.012$ ). The eosinopenia group had significantly higher pro-Brain Natriuretic Peptide (proBNP), D-dimer, neutrophil percentage than the non-eosinopenia group. On the other hand, the eosinopenia group had significantly lower lymphocyte count and lymphocyte percentage. There was no significant difference in baseline $\mathrm{PaO}_{2}$ and $\mathrm{PaCO}_{2}$ between two groups.

\section{Infection severity}

Based on biomarkers of infection, the eosinopenia group consistently presented with more severe infection status than the non-eosinopenia group (Figure 2). The eosinopenia group had significantly higher C-reactive protein (CRP) (median 77.30 vs $16.55, p=0.008$ ) and procalcitonin (median 0.32 vs 0.12 , $\mathrm{p}=0.001)$.

\section{Treatment}


The length of hospital stay was similar between two groups (Table 3 ). But the eosinopenia group had higher hospital cost with statistical significance (median 20598.00 vs $14295.50, p=0.043$ ). There were no significant difference in duration of antibiotic use and use of non-invasive ventilation between the two groups, but there was a clear trend to a higher proportion of invasive ventilation in the eosinopenia group ( $34.1 \%$ vs $11.1 \%, p=0.067$ ). The eosinopenia group had a higher proportion of systemic corticosteroids use $(73.2 \%$ vs $50.0 \%, p=0.083)$, but without statistical significance. Moreover the eosinopenia group had a significantly higher total (median 160.00 vs $40.00, p=0.019$ ) and daily (median 40.00 vs 18.40 , $p=0.028$ ) dose of systemic corticosteroids use. But there was no difference in total and daily dose of inhaled corticosteroids between both two groups.

\section{Long-term mortality}

Kaplan-Meier analyses identified a significant difference between two groups in all-cause mortality after hospital discharge. Survival at 18 months after hospital discharge was significantly lower in the eosinopenia group vs non-eosinopenia group (log rank, $p=0.002$ ) (Figure 3 ).

\section{Discussion}

The potential utility of eosinopenia has not been evaluated in hospitalized AECOPD patients with CAP. The current study showed that eosinopenia $(<50 / \mu \mathrm{L})$ was a strong predictor of 18 -month mortality. In addition, the eosinopenia group had significantly elevated CRP and procalcitonin, which indicated that eosinopenia was associated with more severe infection. Therefore, eosinopenia may be helpful for physicians to identify patients with high risk of mortality and optimize management strategies accordingly. Prospective studies are warranted to evaluate if adopting this strategy could improve prognosis of hospitalized AECOPD patients with CAP.

COPD is one of the most important cause of death worldwide, which was worsened by exacerbations.(2) The CAP occurs commonly in patients with COPD as impairment in lung defense, especially in those older than 65 years.(16) COPD is reported as a comorbidity in at least one third of hospitalized patients with CAP.(4) There is a controversy about the impact of COPD on mortality of patients hospitalized for CAP.(17) Several studies supported that COPD patients with CAP had higher mortality. $(13,18-20)$ However, some studies and meta-analysis showed that mortality was not increased in patients with COPD hospitalized with CAP.(21-23) Regardless of co-existence of CAP, AECOPD caused high mortality worldwide, which warranted attention from physicians. So a simple, accessible and cheap marker to identify those patients with high risk of mortality was helpful in practice, especially in developing countries. Eosinopenia gained our attention, which was further examined in the current study.

Normally, eosinophil account for only a small part of white blood cells in blood. Its roles have been extensively investigated in asthma and COPD. High blood eosinophil count was a reliable predictor of response to inhaled corticosteroid in COPD. (24-27) Furthermore, oral corticosteroid during AECOPD had better effect in patients with high blood eosinophil count.(28) However studies about low blood eosinophil count or eosinopenia was relatively limited. Eosinopenia could be caused by either acute 
infection or acute stress, so it was not a biomarker of infection status. In essence, the eosinopenia of acute infection has been assumed to be a secondary expression of adrenal corticosteroid stimulation produced by the stress of the infection. $(29,30)$ Therefore it was suggested that eosinopenia was a response to the acute inflammatory process rather than to a specific type of pathogen.

The principal finding of the current study was that eosinopenia $(<50 / \mu \mathrm{L})$ was a strong predictor of 18 month mortality. This finding was in agreement with previous studies. Numerous studies supported that eosinopenia was strongly associated with increased mortality in various clinical settings and diseases. In a large retrospective cohort study of 2311 patients with bacteremia, eosinopenia $(<50 / \mu \mathrm{L})$ was associated with a 4.77-fold increase in risk of dying compared with a normal eosinophil count.(31) Yip et al. found that eosinopenia $(<10 / \mu \mathrm{L})$ on ICU discharge was associated with increased post-ICU mortality. (32) Similarly eosinopenia $(<40 / \mu \mathrm{L})$ at ICU admission was reported to be an independent predictor of 28day mortality.(8) Eosinopenia was reported to be able to predict mortality not only in adults but also in children.(5)

A strong association between eosinopenia and mortality in AECOPD had also been described by some studies. A study by Holland et al reported that eosinopenia $(<40 / \mu \mathrm{L})$ was associated with higher inhospital mortality.(10) Moreover eosinopenia was identified as one of five strongest predictors of inpatient mortality in hospitalized AECOPD patients as part of the Dyspnea, Eosinopenia, Consolidation, Acidemia and atrial Fibrillation (DECAF) score.(11) MacDonald et al further demonstrated that eosinopenia $(<50 / \mu \mathrm{L})$ were strongly associated with higher 12-month mortality in hospitalized AECOPD patients. (12) In our dataset, eosinopenia $(<50 / \mu \mathrm{L})$ was associated with increased mortality at 18 months. So our findings corroborated with MacDonald's, but our study population was different from MacDonald's. In our study, only hospitalized AECOPD patients with confirmed CAP were analyzed; in MacDonald's study, hospitalized AECOPD patients with all causes were included. Based on detection of chest X-ray consolidation provided by supplemental data, it was roughly estimated that there were 43 cases of pneumonia out of 242 cases in MacDonald's study. So there were more hospitalized AECOPD patients with CAP in our study than MacDonald's (59 vs 43). Moreover in our study, the diagnosis of CAP was based on chest CT, which was more reliable. But still the study population was relatively small, further studies with a larger population were warranted.

Another important finding of our study was that the eosinopenia group had significantly elevated CRP and procalcitonin. The CRP and procalcitonin were commonly used biomarkers for infection, and procalcitonin is a diagnostic marker of the presence of a bacterially induced systemic inflammatory reaction.(33) Our finding indicated that eosinopenia was associated with more severe infection status. This finding was consistent with previous reports conducted in various clinical settings. The study by Gil et al in a department of internal medicine demonstrated that an inflammatory syndrome associated with eosinopenia $(<40 / \mu \mathrm{L})$ was related to bacterial infectious diseases.(34) Similarly, another study in ICU by Abidi et al showed that eosinopenia was a reliable marker of sepsis, and was associated with the severity of sepsis.(35) Moreover the study in general internal medicine setting, excluding ICU and ED, revealed that eosinopenia was a useful predictor of bloodstream infection.(7) In ED, eosinopenia(< 10/ $\mu \mathrm{L}$ ) was reported 
to present a specificity of $94 \%$ for the diagnosis of infection. As above-mentioned, eosinopenia could be caused by acute stress secondary to both infection and non-infection stimuli. The association between eosinopenia $(<50 / \mu \mathrm{L})$ and higher mortality found in this survey could therefore be partially explained both by more severe infection status and higher stress levels induced by infection.

The current study has a potentially important implication for physicians, especially in developing countries. As a cheap test for early identification of patients at high risk of death on admission, eosinopenia may help physicians in making management strategy. Once identified, a strategy of more aggressive diagnostic and therapeutic interventions may be warranted. The pro and con of such strategy are needed to be verified by prospective cohort studies.

Some limitations of the study merit consideration. First, the present study was a retrospective study performed in a single institution, so there were inherent problems related to this design. Second, patients were identified as having AECOPD by medical history instead of pulmonary function, which has been demonstrated in past studies to be the same method as that used to identify other comorbid conditions included to create the PSI score. The severity of COPD between two groups could not be compared, although the $\mathrm{PaO}_{2}$ and $\mathrm{PaCO}_{2}$ was similar.

In conclusion, eosinopenia $(<50 / \mu \mathrm{L})$ was a strong predictor of 18 -month mortality in hospitalized AECOPD patients with CAP. Moreover eosinopenia was associated with more severe infection. Eosinophil count at admission can be used as a prognosis marker of mortality in hospitalized AECOPD patients with CAP. It may become a helpful clinical tool.

\section{Abbreviations}

COPD: Chronic Obstructive Pulmonary Disease

AECOPD: Acute Exacerbations of Chronic Obstructive Pulmonary Disease

CAP: Community-acquired Pneumonia

ICU: Intensive Care Unit

EMRS: Electric Medical Record System

CT: Computed Tomography

WBC: White Blood Cell

SD: Stand Deviation

IQR: Interquartile Range

ED: Emergency Department 
pro-BNP: pro-Brain Natriuretic Peptide

$\mathrm{PaO}_{2}$ : Arterial Partial Pressure of Oxygen

$\mathrm{PaCO}_{2}$ : Arterial Partial Pressure of Carbon Dioxide

CRP: C-reactive protein

DECAF: Dyspnea, Eosinopenia, Consolidation, Acidemia and atrial Fibrillation

\section{Declarations}

Ethics approval and consent to participate[The study was approved by the Ethics Committee for Human Research of the Second Affiliated Hospital of Zhejiang University School of Medicine (No 2021-0567). Informed consent was obtained for experimentation with human subjects.

Consent for publication[Not applicable.

Availability of data and materialsロThe datasets generated and/or analyzed during the current study are not publicly available due patients' individual privacy could be compromised, but are available from the corresponding author on reasonable request.

Competing interestsםThe authors declare no conflict of interest.

Funding[National Natural Science Foundation of China [NO. 81500061] and Beijing Bethune Charitable Foundation [BJ-RW2020025J].

Authors' contributions $囚 \mathrm{HQH}, \mathrm{YXM}$ and $\mathrm{YYQ}$ designed the study. $Y X \mathrm{XM}, \mathrm{YYQ}, \mathrm{XYS}$ and NL performed the study, collected data and analyzed the data. YXM and NL wrote the paper. All authors critically reviewed the paper and approved it.

\section{References}

1. Labaki WW, Rosenberg SR. Chronic Obstructive Pulmonary Disease. Ann Intern Med. 2020;173(3):Itc17-itc32.

2. MacLeod M, Papi A, Contoli M, Beghé B, Celli BR, Wedzicha JA, et al. Chronic obstructive pulmonary disease exacerbation fundamentals: Diagnosis, treatment, prevention and disease impact. Respirology (Carlton, Vic). 2021;26(6):532-51.

3. Bordon J, Slomka M, Gupta R, Furmanek S, Cavallazzi R, Sethi S, et al. Hospitalization due to community-acquired pneumonia in patients with chronic obstructive pulmonary disease: incidence, epidemiology and outcomes. Clinical microbiology and infection: the official publication of the European Society of Clinical Microbiology and Infectious Diseases. 2020;26(2):220-6. 
4. Cilli A, Erdem H, Karakurt Z, Turkan H, Yazicioglu-Mocin O, Adiguzel N, et al. Community-acquired pneumonia in patients with chronic obstructive pulmonary disease requiring admission to the intensive care unit: risk factors for mortality. Journal of critical care. 2013;28(6):975-9.

5. Kim YH, Park HB, Kim MJ, Kim HS, Lee HS, Han YK, et al. Prognostic usefulness of eosinopenia in the pediatric intensive care unit. Journal of Korean medical science. 2013;28(1):114-9.

6. Al Duhailib Z, Farooqi M, Piticaru J, Alhazzani W, Nair P. The role of eosinophils in sepsis and acute respiratory distress syndrome: a scoping review. Canadian journal of anaesthesia $=$ Journal canadien d'anesthesie. 2021;68(5):715-26.

7. Hirosawa T, Harada Y, Morinaga K, Takase H, Nin M, Shimizu T. Eosinopenia as a diagnostic marker of bloodstream infection in a general internal medicine setting: a cohort study. BMC infectious diseases. 2020;20(1):85.

8. Abidi K, Belayachi J, Derras Y, Khayari ME, Dendane T, Madani N, et al. Eosinopenia, an early marker of increased mortality in critically ill medical patients. Intensive care medicine. 2011;37(7):1136-42.

9. Kolsum U, Donaldson GC, Singh R, Barker BL, Gupta V, George L, et al. Blood and sputum eosinophils in COPD; relationship with bacterial load. Respir Res. 2017;18(1):88.

10. Holland M, Alkhalil M, Chandromouli S, Janjua A, Babores M. Eosinopenia as a marker of mortality and length of stay in patients admitted with exacerbations of chronic obstructive pulmonary disease. Respirology (Carlton, Vic). 2010;15(1):165-7.

11. Steer J, Gibson J, Bourke SC. The DECAF Score: predicting hospital mortality in exacerbations of chronic obstructive pulmonary disease. Thorax. 2012;67(11):970-6.

12. MacDonald MI, Osadnik CR, Bulfin L, Hamza K, Leong P, Wong A, et al. Low and High Blood Eosinophil Counts as Biomarkers in Hospitalized Acute Exacerbations of COPD. Chest. 2019;156(1):92-100.

13. Restrepo MI, Mortensen EM, Pugh JA, Anzueto A. COPD is associated with increased mortality in patients with community-acquired pneumonia. The European respiratory journal. 2006;28(2):34651.

14. Fine MJ, Singer DE, Hanusa BH, Lave JR, Kapoor WN. Validation of a pneumonia prognostic index using the MedisGroups Comparative Hospital Database. The American journal of medicine. 1993;94(2):153-9.

15. Cao B, Huang Y, She DY, Cheng QJ, Fan H, Tian XL, et al. Diagnosis and treatment of communityacquired pneumonia in adults: 2016 clinical practice guidelines by the Chinese Thoracic Society, Chinese Medical Association. The clinical respiratory journal. 2018;12(4):1320-60.

16. Müllerova H, Chigbo C, Hagan GW, Woodhead MA, Miravitlles M, Davis KJ, et al. The natural history of community-acquired pneumonia in COPD patients: a population database analysis. Respiratory medicine. 2012;106(8):1124-33.

17. Cilli A. Community-acquired pneumonia in patients with chronic obstructive pulmonary disease. Current infectious disease reports. 2015;17(1):444. 
18. Lu Z, Cheng Y, Tu X, Chen L, Chen H, Yang J, et al. Community-acquired pneumonia and survival of critically ill acute exacerbation of COPD patients in respiratory intensive care units. Int J Chron Obstruct Pulmon Dis. 2016;11:1867-72.

19. Molinos L, Clemente MG, Miranda B, Alvarez C, del Busto B, Cocina BR, et al. Community-acquired pneumonia in patients with and without chronic obstructive pulmonary disease. The Journal of infection. 2009;58(6):417-24.

20. Shin B, Kim SH, Yong SJ, Lee WY, Park S, Lee SJ, et al. Early readmission and mortality in acute exacerbation of chronic obstructive pulmonary disease with community-acquired pneumonia. Chron Respir Dis. 2019;16:1479972318809480.

21. Snijders D, van der Eerden M, de Graaff C, Boersma W. The influence of COPD on mortality and severity scoring in community-acquired pneumonia. Respiration; international review of thoracic diseases. 2010;79(1):46-53.

22. Loke YK, Kwok CS, Wong JM, Sankaran P, Myint PK. Chronic obstructive pulmonary disease and mortality from pneumonia: meta-analysis. International journal of clinical practice. 2013;67(5):47787.

23. Jiang HL, Chen HX, Liu W, Fan T, Liu GJ, Mao B. Is COPD associated with increased mortality and morbidity in hospitalized pneumonia? A systematic review and meta-analysis. Respirology (Carlton, Vic). 2015;20(7):1046-54.

24. Mycroft K, Krenke R, Górska K. Eosinophils in COPD-Current Concepts and Clinical Implications. The journal of allergy and clinical immunology In practice. 2020;8(8):2565-74.

25. Pascoe S, Locantore N, Dransfield MT, Barnes NC, Pavord ID. Blood eosinophil counts, exacerbations, and response to the addition of inhaled fluticasone furoate to vilanterol in patients with chronic obstructive pulmonary disease: a secondary analysis of data from two parallel randomised controlled trials. Lancet Respir Med. 2015;3(6):435-42.

26. Siddiqui SH, Guasconi A, Vestbo J, Jones P, Agusti A, Paggiaro P, et al. Blood Eosinophils: A Biomarker of Response to Extrafine Beclomethasone/Formoterol in Chronic Obstructive Pulmonary Disease. Am J Respir Crit Care Med. 2015;192(4):523-5.

27. Pavord ID, Lettis S, Locantore N, Pascoe S, Jones PW, Wedzicha JA, et al. Blood eosinophils and inhaled corticosteroid/long-acting $\beta$-2 agonist efficacy in COPD. Thorax. 2016;71(2):118-25.

28. Bafadhel M, McKenna S, Terry S, Mistry V, Pancholi M, Venge P, et al. Blood eosinophils to direct corticosteroid treatment of exacerbations of chronic obstructive pulmonary disease: a randomized placebo-controlled trial. Am J Respir Crit Care Med. 2012;186(1):48-55.

29. Rothenberg ME. Eosinophilia. N Engl J Med. 1998;338(22):1592-600.

30. Bass DA. Behavior of eosinophil leukocytes in acute inflammation. II. Eosinophil dynamics during acute inflammation. The Journal of clinical investigation. 1975;56(4):870-9.

31. Terradas R, Grau S, Blanch J, Riu M, Saballs P, Castells X, et al. Eosinophil count and neutrophillymphocyte count ratio as prognostic markers in patients with bacteremia: a retrospective cohort study. PLoS One. 2012;7(8):e42860. 
32. Yip B, Ho KM. Eosinopenia as a predictor of unexpected re-admission and mortality after intensive care unit discharge. Anaesthesia and intensive care. 2013;41(2):231-41.

33. Titova E, Christensen A, Henriksen AH, Steinshamn S, Åsberg A. Comparison of procalcitonin, Creactive protein, white blood cell count and clinical status in diagnosing pneumonia in patients hospitalized with acute exacerbations of COPD: A prospective observational study. Chron Respir Dis. 2019;16:1479972318769762.

34. Gil H, Magy N, Mauny F, Dupond JL. [Value of eosinopenia in inflammatory disorders: an "old" marker revisited]. La Revue de medecine interne. 2003;24(7):431-5.

35. Abidi K, Khoudri I, Belayachi J, Madani N, Zekraoui A, Zeggwagh AA, et al. Eosinopenia is a reliable marker of sepsis on admission to medical intensive care units. Critical care (London, England). 2008;12(2):R59.

\section{Tables}

Due to technical limitations, table $1,2 \& 3$ is only available as a download in the Supplemental Files section.

\section{Figures}

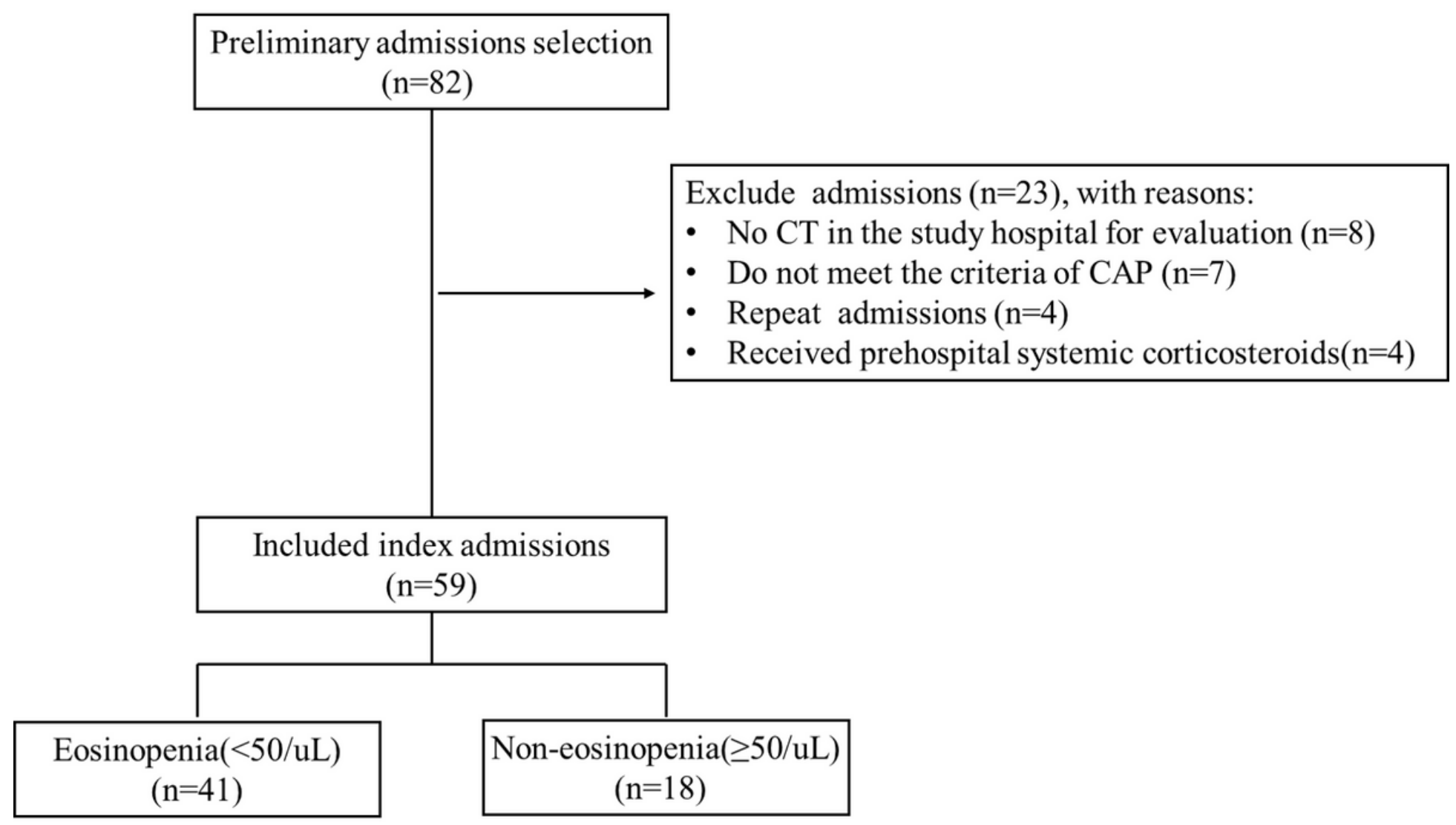

Figure 1 
Flow chart of study population. CT: computed tomography; CAP: community-acquired pneumonia
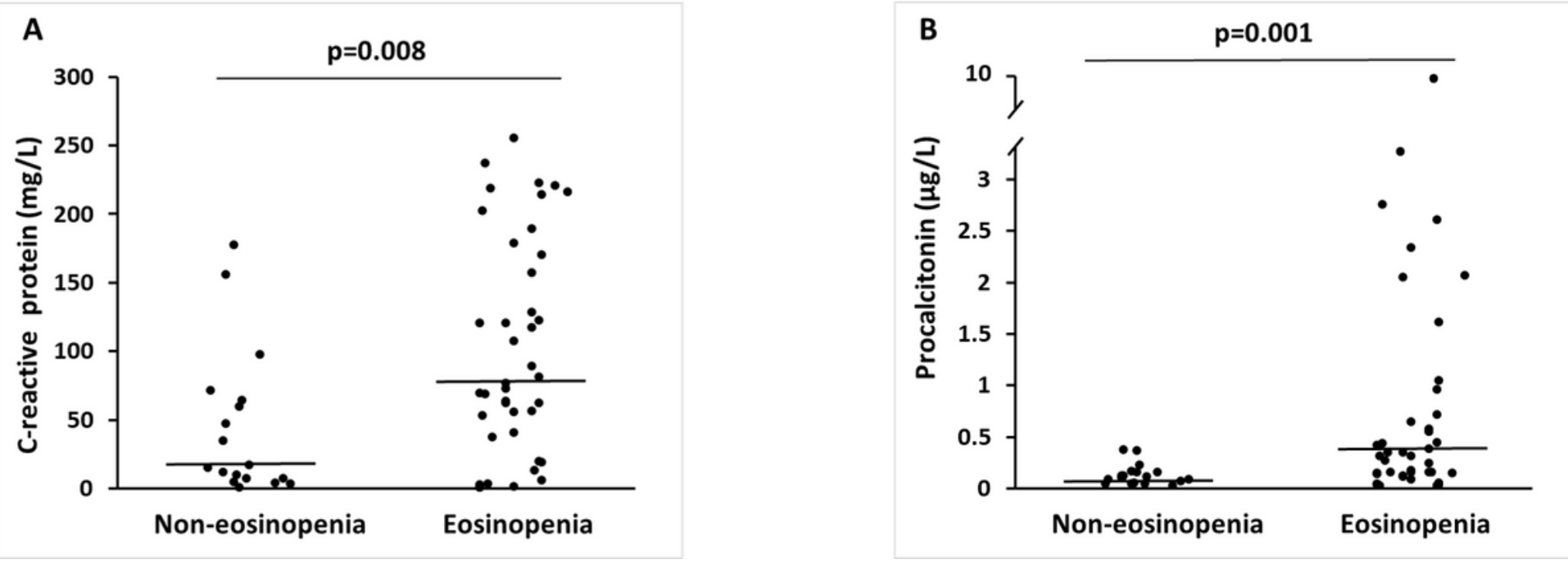

Figure 2

Infection biomarkers between two groups. A, CRP levels; B, procalcitonin levels. CRP: C-reactive protein.

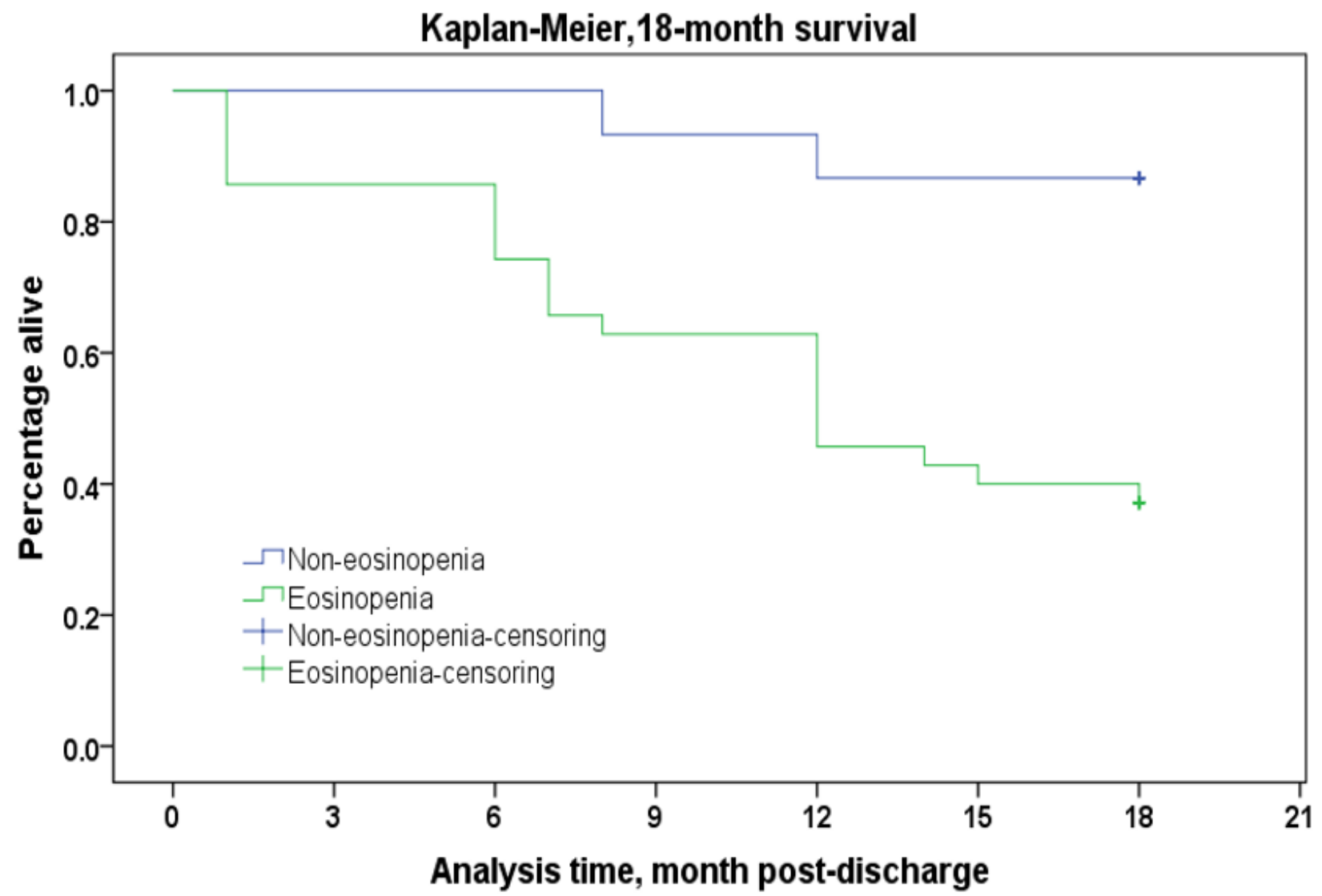

Figure 3 
Eighteen-month survival between two groups. Kaplan-Meier analysis of survival to 18 months after hospital discharge.

\section{Supplementary Files}

This is a list of supplementary files associated with this preprint. Click to download.

- Table1.png

- Table2.png

- Table3.png 